\title{
Clinical Outcomes after Keraring Implantation for Keratoconus Management in Patients Older Than 40 Years: A Retrospective, Interventional, Cohort Study
}

\author{
Zisis Gatzioufas - Aye Khine - Mohamed Elalfy - Ivo Guber • \\ Cameron McLintock · Francesco Sabatino • Samer Hamada • \\ Damian Lake
}

Received: October 10, 2017 / Published online: December 23, 2017

(C) The Author(s) 2017. This article is an open access publication

\section{ABSTRACT}

Background: Intracorneal ring segment implantation is an effective and safe method of visual improvement in patients with keratoconus. The aim of our study was to evaluate the long-term clinical outcomes after Keraring implantation for keratoconus in patients older than 40 years.

Methods: Eleven eyes from 11 patients with keratoconus who underwent femtosecond laserassisted Keraring implantation for keratoconus were included in this retrospective study. The uncorrected visual acuity (UCVA), corrected visual acuity, keratometric readings, central corneal thickness and thinnest corneal pachymetry were evaluated preoperatively and 6 months after the Keraring implantation.

Enhanced content To view enhanced content for this article go to http://www.medengine.com/Redeem/ CFFCF06061440001.

Z. Gatzioufas $(\bowtie) \cdot$ A. Khine · M. Elalfy · I. Guber

C. McLintock · S. Hamada - D. Lake

Corneo-Plastic Unit, Queen Victoria Hospital, East

Grinstead, UK

e-mail: zisisg@hotmail.com

M. Elalfy

The Research Institute of Ophthalmology, Cairo, Egypt

F. Sabatino

Moorfields Eye Hospital, London, UK
Results: UCVA, BCVA and keratometric readings improved at 6 months postoperatively.

Conclusion: Our data showed significant keratometric amelioration and visual improvement after Keraring implantation for keratoconus in patients older than 40 years at 6 months postoperatively.

Keywords: Keraring; Keratoconus; Older patients

\section{INTRODUCTION}

Intracorneal ring segments (ICRS) are small PMMA devices that are implanted into the cornea, aiming to alter its geometry in a manner that will enhance its refractive properties and thereby improve the visual acuity. The mechanism of action in ICRS is simple. They act as spacer elements between the collagen fibers of the corneal stroma and induce an arc-shortening effect resulting in flattening of the central corneal area [1]. Several studies documented that implantation of ICRS decreases the keratometric readings, spherical equivalent and cylinder, reduces high-order aberrations and improves uncorrected distance visual acuity (UDVA) and best-corrected distance visual acuity (BCDVA) in patients with keratoconus [2-6]. The complication rate is relatively low and mostly includes postoperative complications such as infection, ICRS displacement/ 
migration, ICRS extrusion, corneal scarring and corneal vascularization $[6,7]$.

Nowadays there are implantation nomograms indicating the appropriate ring segment features for each individual case. There are also certain indications and contraindications regarding the patient selection to maximize the treatment's safety and efficacy $[6,8]$. However, there are still many 'gray zones' in the preoperative assessment. Which prognostic factors influence the clinical outcome or predict the success/failure rate of this treatment method? The aim of our study was to evaluate the clinical outcomes after Keraring implantation in older patients with keratoconus.

\section{METHODS}

This was a retrospective interventional cohort study including 11 patients with stable keratoconus who underwent unilateral Keraring implantation using the iFS femtosecond laser (Abbott Vision, Abbott Park, IL, USA). All patients had keratoconus stage 2 or 3 according to the Amsler-Krumeich classification [9]. All of the patients had undergone corneal collagen cross-linking in the past and had experienced stable keratoconus for $>12$ months prior to ICRS implantation. Patients were enrolled in the study data set based on the following criteria: age $>40$ years, contact lens intolerance, best-corrected visual acuity $<0.3$ (logMAR) and corneal thickness $>400$ microns in the area of ICRS implantation. In addition, patients had no central corneal scars, ocular surface disease, allergic eye disease, previous ocular surgery or any other ocular pathology except for keratoconus. Pregnant or breast-feeding women were excluded from the study cohort.

A complete ophthalmic examination was performed pre- and postoperatively, including the uncorrected visual acuity (UCVA), best spectacle-corrected visual acuity (BCVA), manifest refraction, spherical equivalent (SE), keratometry $(K)$ readings (in diopters, D), central corneal thickness (in microns, $\mu \mathrm{m}$ ) and thinnest corneal pachymetry (in microns, $\mu \mathrm{m}$ ). Corneal topography was evaluated using a Scheimpflug camera (Pentacam, Oculus Optikgeräte,
Wetzlar, Germany). Diagnosis of keratoconus was facilitated by corneal topography and corneal elevation mapping, as evaluated by Scheimpflug imaging (Pentacam, Oculus Optikgeräte, Wetzlar, Germany). Visual acuity was measured using Snellen notation and then converted to $\log$ MAR for statistical analysis.

The standard nomogram of the ICRS provided by the manufacturers was used for selection of the appropriate ring segment in each individual case and calculation of the implantation parameters. All patients had one ring segment implanted in the flat meridian adjacent to the cone, with 5-mm diameter and variable thickness between 150 and $300 \mu \mathrm{m}$. Intrastromal tunnels were created utilizing the iFS femtosecond laser (Abbott Vision). After topical anesthesia with $0.5 \%$ proparacaine hydrochloride, the corneal apex was marked with ink under a Zeiss OPMI Lumera 700 surgical microscope (Carl Zeiss AG, Jena, Germany). A 9.5-mm suction ring was applied, and after applanation, a corneal tunnel of $1.3-\mathrm{mm}$ width was created with the iFS femtosecond laser in the $5-\mathrm{mm}$ zone and $80 \%$ corneal depth as well as a single radial $2.7-\mathrm{mm}$ corneal incision at the tunnel starting point. The corneal tunnel length was equal to the ring segment arc length plus $10^{\circ}$. Ring segments were inserted into the tunnel with the aid of special forceps. Postoperatively patients were prescribed chloramphenicol $0.5 \%$ eye drops four times a day for 2 weeks and fluorometholone $0.1 \%$ eye drops four times a day for 4 weeks. Scheduled follow-up was at postoperative day 1 and 1 week, 1 month and 6 months postoperatively.

All procedures were followed in accordance with the ethical standards of the responsible committee on human experimentation (institutional and national) and with the Helsinki Declaration of 1964, as revised in 2013. All patients provided their written consent prior to ICRS implantation and the principles of the Declaration of Helsinki were fully respected. The local IRB committee approved this study. The current study was not registered as a clinical trial as this was not required by the ethics committee because of its retrospective nature. 


\section{Statistical Analysis}

Normality of the data distribution was tested using the Shapiro-Wilk test. A paired $t$ test was applied. All results are presented as mean \pm standard deviation. $P<0.05$ was considered statistically significant. All statistical analyses were performed using MedCalc software (version 15; MedCalc, Oostende, Belgium).

\section{RESULTS}

Eleven eyes of 11 patients were included (10 males and 1 female). Mean age was $48.54 \pm 8.43$ (years). Eight patients had keratoconus stage 2, and three patients had keratoconus stage 3 according to the Amsler-Krumeich classification. All treated eyes had undergone corneal cross-linking treatment in the past. The vast majority of patients (10/11) were suffering from contact lens intolerance. Demographic data are presented in Table 1.

Significant improvement was observed in all parameters examined in this study between baseline and follow-up at 6 months apart from the CCT and corneal thinnest point. Specifically, corrected and uncorrected distance visual acuity significantly increased, whereas spherical equivalent, corneal astigmatism, Kmax and Kmean significantly decreased 6 months postoperatively (all $P<0.05$ ). All results are summarized in Table 2. No complications occurred.

Table 1 Demographic data of the study group

\begin{tabular}{ll}
\hline Number of patients & 11 \\
Mean age \pm SD & $48.54 \pm 8.43$ (years) \\
Male:female ratio & $10: 1$ \\
Right eye:left eye ratio & $6: 5$ \\
KC stage 2:KC stage 3 ratio & $8: 3$ \\
Contact lens intolerance & 10 \\
Previous corneal cross-linking & 11 \\
\hline
\end{tabular}

$K C$ keratoconus
Table 2 Summary of the visual, refractive, corneal topographic and tomographic outcomes after Keraring implantation for keratoconus in patients older than 40 years

\begin{tabular}{lccl}
\hline & Pre-op & Post-op & \multicolumn{1}{l}{$\boldsymbol{P}$} \\
\hline $\begin{array}{l}\text { UDVA } \\
(\operatorname{logMAR})\end{array}$ & $0.94 \pm 0.1$ & $0.76 \pm 0.28$ & 0.01 \\
$\begin{array}{l}\text { CDVA } \\
(\text { logMAR })\end{array}$ & $0.41 \pm 0.27$ & $0.2 \pm 0.2$ & 0.01 \\
$\begin{array}{l}\text { Spherical } \\
\text { equivalent }\end{array}$ & $-11.65 \pm 4.48$ & $-4.44 \pm 3.9$ & 0.005 \\
$\quad(D)$ & & & \\
$\begin{array}{l}\text { Corneal } \\
\text { astigmatism }\end{array}$ & $-4.48 \pm 3.74$ & $-2.75 \pm 2.18$ & 0.01 \\
$\quad(D)$ & & & \\
Kmax $(D)$ & $63.24 \pm 10.57$ & $60.15 \pm 6.25$ & 0.02 \\
Kmean $(D)$ & $51.40 \pm 5.50$ & $48.43 \pm 5.28$ & 0.02 \\
CCT $(\mu \mathrm{m})$ & $466.54 \pm 38.8$ & $472.9 \pm 37.5$ & 0.3 \\
Corneal & $449.0 \pm 38.54$ & $459.3 \pm 38.7$ & 0.2 \\
thinnest & & & \\
point $(\mu \mathrm{m})$ & & & \\
\hline
\end{tabular}

Pre-op preoperatively, Post-op postoperatively, UDVA uncorrected distance visual acuity, CDVA corrected distance visual acuity, Kmax maximal keratometry, Kmean mean keratometry, $C C T$ central corneal thickness, CTP corneal thinnest point. $p<0.05$ was considered statistically significant

\section{DISCUSSION}

Intracorneal ring segments act as space elements between the collagen fibers in the corneal stroma, thereby inducing an arcshortening effect that results in flattening of the central cornea [1]. There is evidence that their flattening effect is directly proportional to the thickness of the implanted segment and inversely proportional to the corneal diameter at the implantation site [10]. The efficacy of ICRS in the management of keratoconus has been well documented. ICRS reduce spherical equivalent and keratometric readings, as well as high-order aberrations and particularly coma, thereby 
improving UCVA and CDVA $[2,3,8,11]$. The increase in visual acuity is partly attributed to the improvement of the aberrometric profile but mainly to the reduction of coma as a result of the regularized corneal geometry after ICRS implantation [3, 12]. Moreover, ICRS implantation improved the contact lens tolerance significantly, as shown by numerous studies $[11,13,14]$.

Several factors associated with the visual outcomes after ICRS implantation have been identified. Vega-Estrada et al. reported that poor preoperative visual acuity is a good prognostic factor for significant visual improvement [3], whereas Alio et al. suggested that it is less likely to achieve significant visual improvement in advanced keratoconus (stage 4) [15]. Advanced keratoconus has also been linked to low predictability of the keratometric and visual outcomes after ICRS implantation [16]. Alio et al. proposed that alignment of the refractive and keratometric axes $\left(\right.$ angle $<15^{\circ}$ ) is another positive prognostic factor for successful visual outcomes after ICRS implantation [17].

The aim of our study was to evaluate the clinical outcomes after Keraring implantation for keratoconus in patients older than 40 years. It has been documented that corneal stiffness increases with age, mimicking the corneal stiffening effect of corneal collagen cross-linking $[18,19]$. ICRS act as space elements between the collagen fibers in the corneal stroma and therefore their functional efficacy could be compromised in older patients who have stiffer corneas. However, the present study was not designed to evaluate whether age-related corneal changes or corneal cross-linking-induced changes inhibited the progression of corneal ectasia in the study population and stabilized their keratometric measurements. Therefore, we cannot comment on the exact role of age or corneal cross-linking in stabilizing corneal ectasia in older patients with keratoconus. Our study only investigated the clinical efficacy and safety of Keraring implantation in patients with keratoconus older than 40 years, showing that there was improvement of UCVA, BCVA and keratometric readings. Of course, our study has certain limitations. The number of eyes included in our study was low. No intra-group comparisons were possible because of the low number of eyes. The inherent potential for selection bias should also be considered in our retrospective study. However, all patients who underwent ICRS implantation and met the inclusion and exclusion criteria were included in the study. The dropout rate for this study was zero and all patients were included in the analysis through the study follow-up without retention or loss of subject data. Of course, there might be a higher age threshold for reduced efficacy of ICRS, which our study was not able to identify.

\section{CONCLUSIONS}

In conclusion, our data show that implantation of Kerarings in patient with keratoconus older than 40 years is both effective and safe. Larger comparative studies are required however to further investigate the potential role of age in visual outcomes after ICRS implantation for keratoconus.

\section{ACKNOWLEDGEMENTS}

Funding. No funding or sponsorship was received for this study or publication of this article.

Authorship. All named authors meet the International Committee of Medical Journal Editors (ICMJE) criteria for authorship for this manuscript, take responsibility for the integrity of the work as a whole and have given final approval for the version to be published.

Disclosures. Zisis Gatzioufas, Aye Khine, Mohamed Elalfy, Ivo Guber, Cameron McLintock, Francesco Sabatino, Samer Hamada and Damian Lake have nothing to disclose.

Compliance with Ethics Guidelines. All procedures followed were in accordance with the ethical standards of the responsible committee on human experimentation (institutional and national) and with the Helsinki 
Declaration of 1964, as revised in 2013. All patients provided their written consent prior to ICRS implantation and the principles of the Declaration of Helsinki were fully respected. The local IRB committee approved this study.

Data availability. The datasets during and/ or analysed during the current study are available from the corresponding author on reasonable request.

Open Access. This article is distributed under the terms of the Creative Commons Attribution-NonCommercial 4.0 International License (http://creativecommons.org/licenses/ by-nc/4.0/), which permits any noncommercial use, distribution, and reproduction in any medium, provided you give appropriate credit to the original author(s) and the source, provide a link to the Creative Commons license, and indicate if changes were made.

\section{REFERENCES}

1. Silvestrini TA, Mathis ML, Loomas BE, Burris TE. A geometric model to predict the change in corneal curvature from the intrastromal corneal ring (ICR). Investig Ophthalmol Vis Sci. 1994;35:2023.

2. Piñero DP, Alio JL. Intracorneal ring segments in ectatic corneal disease-a review. Clin Exp Ophthalmol. 2010;38(2):154-67.

3. Vega-Estrada A, Alio JL, Brenner LF, Javaloy J, Plaza Puche AB, Barraquer RI, et al. Outcome analysis of intracorneal ring segments for the treatment of keratoconus based on visual, refractive, and aberrometric impairment. Am J Ophthalmol. 2013;155(3):575-84.

4. Coskunseven E, Kymionis GD, Tsiklis NS, Atun S, Arslan E, Jankov MR, Pallikaris IG. One-year results of intrastromal corneal ring segment implantation (KeraRing) using femtosecondlaser in patients with keratoconus. Am J Ophthalmol. 2008;145(5):775-9.

5. Kaya V, Utine CA, Karakus SH, Kavadarli I, Yilmaz OF. Refractive and visual outcomes after Intacs vs ferrara intrastromal corneal ring segment implantation for keratoconus: a comparative study. J Refract Surg. 2011;27(12):907-12.
6. Giacomin NT, Mello GR, Medeiros CS, Kiliç A, Serpe CC, Almeida HG, Kara-Junior N, Santhiago MR. Intracorneal ring segments implantation for corneal ectasia. J Refract Surg. 2016;32(12):829-39.

7. Coskunseven E, Kymionis GD, Tsiklis NS, Atun S, Arslan E, Siganos CS, Jankov M, Pallikaris IG. Complications of intrastromal corneal ring segment implantation using a femtosecond laser for channel creation: a survey of 850 eyes with keratoconus. Acta Ophthalmol. 2011;89(1):54-7.

8. Vega-Estrada A, Alio JL. The use of intracorneal ring segments in keratoconus. Eye Vis (Lond). 2016;3:8.

9. Krachmer JH, Feder RS, Belin MW. Keratoconus and related non-inflammatory corneal thinning disorders. Surv Ophthalmol. 1984;28:293-322.

10. Burris TE, Baker PC, Ayer CT, Loomas BE, Mathis $\mathrm{ML}$, Silvestrini TA. Flattening of central corneal curvature with intrastromal corneal rings of increasing thickness: an eye-bank eye study. J Cataract Refract Surg. 1993;19:182-7.

11. Alió JL, Shabayek MH, Artola A. Intracorneal ring segments for keratoconus correction: long-term follow-up. J Cataract Refract Surg. 2006;32:978-85.

12. Vega-Estrada A, Alió JL, Brenner LF, Burguera N. Outcomes of intrastromal corneal ring segments for treatment of keratoconus: five-year follow-up analysis. J Cataract Refract Surg. 2013;39:1234-40.

13. Carrasquillo KG, Rand J, Talamo JH. Intacs for keratoconus and post-LASIK ectasia: mechanical versus femtosecond laser-assisted channel creation. Cornea. 2007;26:956-62.

14. Shetty R, Kurian M, Anand D, Mhaske P, Narayana $\mathrm{KM}$, Shetty BK. Intacs in advanced keratoconus. Cornea. 2008;27:1022-9.

15. Alió JL, Shabayek MH, Belda JI, Correas P, Diez Feijoo E. Analysis of results related to good and bad outcomes of Intacs implantation for keratoconus correction. J Cataract Refract Surg. 2006;32:756-61.

16. Zare MA, Hashemi H, Salari MR. Intracorneal ring segment implantation for the management of keratoconus: safety and efficacy. J Cataract Refract Surg. 2007;33:1886-91.

17. Peña-García P, Alió JL, Vega-Estrada A, Barraquer RI. Internal, corneal, and refractive astigmatism as prognostic factors for intrastromal corneal ring segment implantation in mild to moderate keratoconus. J Cataract Refract Surg. 2014;40(10):1633-44. 
18. Kida T, Liu JH, Weinreb RN. Effects of aging on corneal biomechanical properties and their impact on 24-h measurement of intraocular pressure. Am J Ophthalmol. 2008;146(4):567-72.
19. Elsheikh A, Wang D, Brown M, Rama P, Campanelli M, Pye D. Assessment of corneal biomechanical properties and their variation with age. Curr Eye Res. 2007;32(1):11-9. 